NASA Techn;:al Memorandum 87003

\title{
Numerical Calculation of Subsonic Jets in Crossflow with Reduced Numerical Diffusion
}

Russell W. Claus

Lewis Research Center

Cleveland, Ohio

Prepared for the

Twenty-first Joint Propulsion Conference cosponsored by the AIAA, SAE, and ASME Monterey, California, July 8-10, 1985

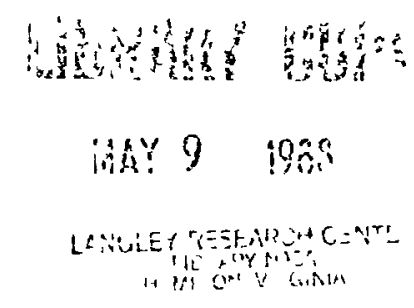


Russell W. Claus

National Aeronautics and Space Administration

Lewis Research Center

Cleveland, Ohro 44135

\section{Abstract}

A series of calculations are reported for two, experimentally studied, subsonic jet in crossflow geometries. The parametric variation examined involves the lateral spacing of a row of jets. The first series of calculations corresponds to a widely spaced jet geometry, $S / O=4$, and the $\stackrel{\sim}{\sim}$ second series corresponds to closely spaced jets,

' $S / D=2$. The calculations are done with alternate differencing schemes to illustrate the impact of numerical diffusion. The calculated jet trajectories agreed well with experimental data in the widely spaced jet geometry, but not in the closely spaced geometry.

\section{Introduction}

In gas turbine engines the mixing of jets in crossflow plays a dominant role in establishing the temperature profile leaving the combustor. This temperature profile, in turn, significantly affects the durability of the turbine. It is the process of tailoring this temperature profile into a pattern acceptable to the turbine that usually consumes the greatest amount of design and development testing in the evolution of a new combustion system.

To reduce this design and development time (1.e., expense) combustor designers currently use empirical correlations to design rows of jets in the combustor wall that will provide an acceptable temperature profile. The difficulty arises due to the limited applicability of these correlations. Empirical correlations are constructed from "idealized" flow field experiments. The flow fields in practical combustion systems exhibit severe nonuniformities in the velocity and temperature fields into which the jets are injected. Empirical correlations can be used in these situations only at great risk.

With the increased capabilities of current computing systems, a much more promising approach is to employ numerical flow codes in the design process. This approach also involves some risk since current combustor flow codes have not been fully verified against most of the complex flow freld features occurring within the combustor. Indeed as noted by Kenworthy et al., 2 the deficiencies of current computer codes make the prediction of combustor exit temperature profiles untenable.

Currently there are two main factors limiting the predictive accuracy of combustor flow codes. First, the proper physics must be represented in the equations solved by the numerical code. The actual physics removed in the large number of modeling assumptions may severely restrict the usefuliness of the code. Second, the numerical accuracy of these codes must be improved.
Current computer codes employ upwind differencing which can introduce an appreciable error in the calculated results. This error (or numerical diffusion) is frequently of such a large magnitude that it swamps or obsecures the turbulence model used in the calculation. Studies under the Hot Section Technology (HOST) aerothermal modeling program identified this error as being one of the key "bottlenecks" to the development of improyed physical submodels in combustor flow codes. $2-4$

Many previous numerical studies $2,5-7$ have examined jets in crossflow. These calculations have employed relatively coarse meshes that could not adequately resolve the flow fieid.' The numerical diffusion in these calcuiations makes the solution strongly grid-dependent. Hence, any agreement or disagreement with experimental data may be more associated with the number of grid points used in the calculation than a test of model assumptions.

The purpose of this report is to provide a clearer distinction between the error associated with numerical diffusion and the error associated with turbulence model assumptions. A series of calculations are made for two different jet in crossflow geometries, widely spaced jets and closely spaced jets. These calculations are made using two alternate forms of convective differencing, Bounded Skew Upwind Differencing (BSUDS2) and the more commonly used hybrid differencing. By making these calculations on relatively fine computational meshes (either 55 by 29 by 12 or 55 by 29 by 10 grid points) and using differencing schemes of varying accuracy, the distinction between turbulence modeling deficiencies and numerical accuracy could be illustrated. The results of the calculations indicate that for the widely spaced jet geometry the penetration of the jet could be well predicted. In the closely spaced jet geometry, the pentration of the jet was found to be sensitive to the specification of the boundary condition.

\section{Symbol List}

$C_{\mu}, C_{\varepsilon l}, C_{\varepsilon 2}$ turbulence model constants

D jet outlet diameter

$k \quad$ turbulençe kinetic energy

$=1 / 2\left(u^{\prime 2}+v^{\prime 2}+w^{2}\right)$

P pressure

$\mathrm{Pe}_{\mathrm{C}} \quad$ cell Peclet number

S source term

$U_{1} \quad$ mean velocity

$u_{1} \quad$ fluctuating velocity about mean 


$$
\begin{array}{ll}
u^{\prime}, v^{\prime}, w^{\prime} & \begin{array}{l}
\text { rms turbulent intensity in } x, y, z \\
\text { direction, respectively }
\end{array} \\
v & \text { mean vertical (y) velocity } \\
\varepsilon & \text { turbulence energy dissipation rate } \\
\mu & \text { viscosity } \\
\rho & \text { density } \\
\sigma & \text { Schmidt number } \\
\varphi & \text { any of the independent variables }
\end{array}
$$

Subscripts:

$\begin{array}{ll}\mathrm{J} & \text { jet condition (average) } \\ \mathrm{P} & \text { at point } P \\ \mathrm{t} & \text { turbulent } \\ \infty & \text { corresponding to mainstream (average) } \\ & \text { Mathematical Formulation }\end{array}$

The numerical code employed in this study was developed under NASA funding and provides the capability to analyze steady-state, three-dimensional, elliptic turbulent flows. Oniy the pertinent features of the code shall be reviewed here as further detalls are avallable in the open literature.

The governing equations, written in tensor notation, include:

\section{Continurty}

$$
\frac{\partial U_{1}}{\partial x_{U}}=0
$$

Momentum

$$
\underbrace{U_{1} \frac{\partial U_{1}}{\partial x_{j}}=}_{\text {Convection }} \underbrace{-\frac{1}{\rho} \frac{\partial p}{\partial x_{j}}+\frac{\partial}{\partial x_{1}}\left(\frac{\mu}{\rho} \frac{\partial U_{j}}{\partial x_{1}}-\overline{u_{1} u_{j}}\right)}_{\begin{array}{c}
\text { Pressure } \\
\text { gradient }
\end{array}}
$$

\section{Scalar transport}

$$
\frac{U_{1} \frac{\partial \varphi}{\partial x_{1}}=\frac{1}{\rho}\left(\frac{\partial}{\partial x_{1}} \frac{\mu}{\sigma_{t}} \frac{\partial \varphi}{\partial x_{1}}-\overline{u_{1} \varphi}\right)}{\text { Convection }}
$$

Turbulence model

$$
\begin{gathered}
\overline{u_{1} u_{J}}=\frac{2}{3} \frac{\delta v_{j}}{\rho} k-\frac{\mu_{t}}{\rho}\left(\frac{\partial U_{1}}{\partial x_{j}}+\frac{\partial U_{j}}{\partial x_{1}}\right) \\
\mu_{t}=C_{\mu} \rho \frac{k^{2}}{\varepsilon} \\
\overline{u_{1} \varphi}=-\frac{1}{\rho} \frac{u_{t}}{\sigma_{t}} \frac{\partial \varphi}{\partial x_{1}}
\end{gathered}
$$

$$
\begin{gathered}
U_{1} \frac{\partial k}{\partial x_{1}}=\frac{1}{\rho} \frac{\partial}{\partial x_{1}}\left(\frac{\mu_{t}}{\sigma_{k}} \frac{\partial_{k}}{\partial x_{1}}\right)-\overline{u_{1} u_{j}} \frac{\partial U_{J}}{\partial x_{1}}-\varepsilon \\
U_{1} \frac{\partial \varepsilon}{\partial x_{1}}=\frac{1}{\rho} \frac{\partial}{\partial x_{1}}\left(\frac{\mu_{t}}{\sigma_{\varepsilon}} \frac{\partial \varepsilon}{\partial x_{1}}\right)-C_{\varepsilon_{1}} \frac{\varepsilon}{k} \overline{u_{1} u_{J}}-C_{\varepsilon_{2}} \frac{\varepsilon^{2}}{k}
\end{gathered}
$$

The turbulence mode $1^{9}$ used represents a two equation level of closure wherein differential equations for the turbulence kinetic energy and turbulence dissipation are solved. The major modeling assumption employed is the characterization of turbulence as being isotropic. The empirical constants used in the equations are the values recommended in Ref. 9 .

The governing equations are discretized through integral analysis or the "finite volume" method. Integrals across each computational cell face are evaluated using the Mean value Theorem on the grid 1 llustrated in Fig. 1 . In effect this procedure is equivalent to finite differencing of the partial differential Eqs. 2 to 5 . The pressure gradient and diffusive terms are approximated using central differences. The convective terms were approximated using either hybrid or BSUDS2 differencing as discussed in the following section. The resulting fluxes across each control volume face can be summed and arranged in a substitution formula. For example, the substitution formula for $\varphi p$ becomes:

$$
\varphi_{p}=\sum_{n b} \phi_{i}+s
$$

where $\sum_{n b}$ denotes summation of the neighbors

of $P$. The system of equations represented by the above was solved using a TriDiagonal Matrix Algorithm (TDMA) procedure sweeping in alternate directions (ADI).

\section{Convectuve Differencing}

Hybrid differencing. The practice most commonly used, at present, is to emp loy hybrid differencing to approximate the convective terms. This involves the use of second order accurate central differencing when the absolute value of the cell Peclet number $\left(\mathrm{Pe}_{\mathrm{C}}\right)$ is less than two, while first order accurate upwind differencing is used when $|\mathrm{Pec}|>2$. The major advantage of this scheme is the unconditionally bounded solutions it provides. A bounded solution is a solution free of nonphysical oscillations. On the contary, an unbounded solution can provide physically unrealistic oscillations or wiggles in the computed result.

Bounded Skew Upwind Differencing Scheme 2 (BSUDS2). BSUDS2 is a derivative of the skewupwind dfferencing (SUD) scheme developed by Raithby. 10 SUD is formally, first order accurate and is not unconditionally bounded. While SUD is formally the same order accuracy as upwind differencing, its truncation error is less than upwind. SUD attains this higher accuracy by differencing in an upwind manner along the flow streamlines. Each streamline is defined by the velocity vector at each grid boundary. The upstream value of the variable to be calculated is then obtained by a 
back projection of the velocity vector and simply interpolating between the neighboring values. The three-dimensional interpolation involves a 27 point computational molecule, (Fig. 1).

While the accuracy of SUD is formaliy first order, it has displayed second order accuracy in a
number of scalar transport test calculations. When SUD is applied to the momentum equations, its accuracy may be lessened due to a fallure to account for source terms in the equation. In other words, a strong pressure gradient (source term in the momentum equations) may invaindate the linear interpolation of velocity at each compytational cell face. Laminar test calculations, 11 indicate that in some flow fields this may compromise accuracy. However, in all calculations SUD was generally at least as accurate as hybrid differencing and, for flow fields having some similarity with jets-in-crossflow, it was much more accurate.

The concept of flux-blending is analogous to the "Flux Corrected Transport" (FCT) technique of Borls and Book. The procedures employed here were developed by Gosman, Lar, and Peric and are detalled in Ref. 8. In general, the flux blended schemes employ a werghted mean of a bounded (but low order accuracy) differencing scheme and an unbounded, more accurate scheme. The main factor is to blend as little of the lesser accurate scheme as possible while still maintaining a properly bounded solution. The two differencing schemes blended involve upwind differencing and the more accurate SUD.

"Bounded Skew Upwind Differencing Scheme 2" (BSUDS2) blends upwind and SUD in proportions ensuring that when negative coefficients occur, their contribution is below the level that would cause the solution to be physically unrealistic. This procedure is iterative and starts from an initial, totally skew differenced estimate. If the calculated variable has a value no greater or lesser than that of its neighbors, then the solution is bounded and no blending is performed. If the solution is out of the range of neighboring values, then blending is performed. In the extreme, this blending would result in upwind differencing. The use of neighboring values as limits in determining the "boundedness" of the solution is only valid when the equation being solved lacks source terms. However, the momentum equations contain significant source contributions. The implications of this are still bejng studied, but the results previously reported demonstrate the use of neighboring values as physical limits provides highly accurate results.

This bounding procedure, while simple in concept, is difficult to apply to an iterative solution scheme. If an initial SUD calculation was made and then the coefficients were updated for bounding and the equation solved a second $t$ ime, the computational time required for one iteration would be approximately doubled. To reduce this computational overhead BSUDS2 calculations were typically restarted from a hybrid calculation with the bounding evaluated based on the previous iteration values. This results in some "unboundedness" when the equations are not fully converged, however, the final result is bounded.
There are two main factors that differ between the implementation of BSUDS2 in Ref. 8 and the procedure used here. First, the bounding factors were calculated for each iteration as opposed to the relaxation procedure of Ref. 8. This slightiy slowed convergence, but eliminated any path dependency or "unboundedness" problems. Second, BSUDS2 calculations were always started from a hybrid solution to improve the stability of the calculation. In fact, the initial BSUDS2 itera$t$ ions employed a switch that set the calculation to hybrid differencing at any point in the flow field that was unbounded. This greatly improved stability at no loss in accuracy, since the final few iterations fully utilized the BSUDS2 procedure.

\section{Solution Algorithm}

The solution algorithm for solving the governing equations will be only briefly reviewed here, Refs. 8 and 13 are recommended for further getails.

Once the momentum equations are approximated on the staggered grid, these equations must be soived in a process insuring that continuity is satisfied. In the SIMPLE algorithm, each momentum equation is sequentially solved using a guessed or old pressure field from the previous iteration. A pressure correction equation is then solved and the values of velocity and pressure are revised to more closely satisfy continuity. Following this, the turbulence and scalar equations are solved using the new velocity values. Iteration on this is then continued until all equations are satisfied to a low normalized residual level (typically $10^{-2}$ for the calculations herein reported).

Calculations were initiated using Pressure Implifft Split Operator (PISO) as developed by Issa. 14 This procedure more closely satisfies continurty for each interation of the momentum equations. It involves a secondary correction that is performed after the pressure and velocity correction used in the SIMPLE procedure. This secondary correction accounts for terms ignored in the SIMPLE procedure. The neglected terms have no effect on the final converged solution, but including them in the first few jpterations can significantly speed convergence. ${ }^{15}$ Because PISO requires almost twice as much computational effort as SIMPLE, when the solution was close to convergence the secondary correction was avoided and the solution procedure reverted to SIMPLE.

Boundary Conditions and Computational Mesh

For all of the calculations reported here, the coordinate system originates at the center of the jet outlet as shown in Fig. 2. The other pertinent geometric parameters are detalled in Fig. 2 .

Both series of calculations used comparably fine computational meshes. The widely spaced calculations used 55 by 29 by 12 grid points in the $x, y, z$ directions respectively. The closely spaced jet calculations used 55 by 29 by 10 grid points. To improve the resolution of the velocity gradients, the grid points were axially clustered near the jet outlet as displayed in the velocity vector plot of Fig. 3 . 
Inflow boundary conditions pose a problem for the jet in crossflow geometry. The upstream boundary condition for the crossflow can be imposed sufficiently far upstream $(X / D=-4)$ to avold any effect on the calculation. The outlet of the penetrating jet is usually treated by adjusting the boundary conditions (velocity profile) to correspond to the measurements of Andreopoulos.16 However, these measurements are for a single jet in crossflow with no restriction in the lateral (Z) direction. By moving the jets closer together (such as in the calculations reported herein) the use of this profile becomes increasingly more suspect. To eliminate this uncertainty from the parametric variation, both the widely spaced $(S / D=4)$ and the closely spaced $(S / D=2)$ jet calculations specified uniform jet profiles two jet diameters upsteam of the jet injection point as seen in Fig. 2.

The jet outlet (or supply pipe) was geometrically approximated as shown in Fig. 4. Calculations were also performed using equal area rectangular slots to test the sensitivity of this geometric approximation. The shape of the outlet had only a small effect on the calculated results. This is consistent with the experimental findings of Holdeman et al. ${ }^{2}$ Holdeman studied jet mixing for both round and square orifices. The controlling parameter appeared to be the jet-tomainstream momentum flux ratio, whereas the shape of the orifice had only a small effect on the jet mixing.

\section{Results and Discussion}

A series of calculations are reported here for two of the geometries experimentally studied by Khan. 7 The parametric variation examined here involves the lateral spacing of a row of jets with a constant jet-to-mainstream velocity ratio $\left(V_{J} / U_{\infty}\right)$ of 2.3 .

Widely spaced jets, $S / D=4$. Numerical calculations of widely spaced jets are compared to the experimental measurements of Khan, Crabb et al., Io and Andreopoulos.16 The latter two experiments are for a single, unconfined jet in crossflow as aistinct from the row of jets configuration of Khan. The calculations reported here are for the row of jets geometry, therefore, the comparisons with the single jet experiments must be made only in regions of the flow freld that are not very sensitive to this variation. This expediency is required since Khan's experiments do not provide the full range of data needed for a complete comparison. This comparison is likely to be most appropriate along the jet centerplane $(Z / D=0)$, near the jet outlet $(X / D=0)$.

Velocity $(V / V J)$ and turbulence intensity $\left(V^{\prime} / V_{j}\right)$ profiles at the jet outlet along the centerline are shown in Fig. 5. As noted above, the experimental data is for a single, unconfined jet, but this is unlikely to effect the comparison at this point in the flow field. The velocity profiles calculated using either hybrid differencing or BSUDS2 are influenced by the crossflow. The forward portion of the jet is decelerated and the downstream portion of the jet is accelerated. Both hybrid and BSUDS2 qualitatively follow this trend, but neither exactly agrees with exper1mental data. The BSUDS2 calculations are more strongly decelerated at the front of the jet and more strongly accelerated at the rear of the jet than either the hybrid results or experimental data. The greater distortion seen in the BSUDS2 calculations indicates that the numerical diffusion provided by hybrid differencing yields a sort of "buffer" which limits the influence of the crossflow. This is consistent with the findings of Ref. 19, wherein the approximated numerical diffusion error increased greatly in magnitude along the edges of the jet. The BSUDS2 calculations are less affected by numerical diffusion and, therefore, more strongly influenced by the crossflow.

The turbulence intensities, (Fig. $5(b))$, are only slightly different for the hybrid and BSUDS2 calculations. Both calculations generaliy underpredict the turbulence intensities experimentally measured. This is especially true near the edges of the jet outlet where experimental values peaked around 25 percent versus the calculated peak of 10 percent. Andreopoulos experimentally observed large scale structures in the flow field near these points. Possibly this accounts for the large discrepancy seen in the turbulence levels. The plenthora of assumptions involved in the $k, \varepsilon$ turbulence model $c$ an be violated under these flow field conditions.

This underprediction of turbulence levels at the jet outlet may not be significant to the jet development external to the outlet. The severe velocity gradients in this external region can locally cause the generation terms in the $k$ equation to dominate over the other terms (transport) in the equation. This was indicated by the relative insensitivity of the $k$ and $\varepsilon$ equations to the form of convective differencing. When the momentum equations were solved using BSUDS2, the turbulence levels computed using either BSUDS2 or hybrid differencing for the $k$ and $\varepsilon$ equations were relatively uneffected. With the more accurate BSUDS2, the computed turbulence levels displayed siightly higher peak levels, but the variation was not great. The turbulence quantities, $k$ and $\varepsilon$, were iargely determined by the source generation terms in the regions of steep velocity gradients.

External to the jet outlet there was a marked difference in the velocity gradients between BSUDS2 and hybrid calculations and this, in turn, strongly impacted the computed turbulence intensities. Support of this statement can be seen in Fig. 6. The turbulence intensities along the jet centerplane at 1.35 jet diameters above the outlet are very sensitive to the form of differencing employed on the momentum equations. The steeper velocity gradients calculated by BSUDS2 leads to both higher turbulence intensities and a better comparison with experiment than the hybrid calculations. The BSUDS2 intensities follow the experimental trend of displaying peak intensities at both $X / D=0$ and 1 . Although the second peak at $X / D=1$ is significantiy underpredicted, the trend agreement is encouraging. The hybrid calculations display a peak intensity at the wrong location and generally show lower intensities than either BSUUS2 or experiment. 
The somewhat favorable agreement between experiment and the BSUDS2 calculations should be observed with caution. A jet in crossflow produces a severely anisotropic turbulence field. The measurements of Crabb display a lateral turbulence intensity ( $w^{\prime}$ in $z$ direction) that is a third larger than the axial intensities ( $u^{\prime}$ ) along the jet centerplane at $x / 0=8$. Closer to the jet outlet as shown in Fig. 6, this anisotropy is likely to be even more significant. Experimental support for this (albeit in different flows) can be found in Refs. 20 and 21. Lateral intensities were not reported at the same location as the axial intensities of $F i g$. 6 , but it seems safe to assume that the isotropic $k, \varepsilon$ turbulence model significantly unoerpredicts the lateral intensities.

The steeper velocity gradients in the BSUDS2 calculations are evident in the centerline axial velocity profiles of Fig. 7. In these profiles, the magnitude of the peak velocity at $Y / D=3.0$ in Fig. $7(\mathrm{a})$ and $Y / D=3.5$ in Fig. $7(\mathrm{~b})$ is underpredicted using hybrid differencing and overpredicted using BSUDS2. An overprediction of the velocity peak would be expected from the underprediction of turbulence intensities seen in Fig. 6. Only the BSUDS2 calculations are sufficientiy free of numerical diffusion to provide physically consistent results. The hybrid results uncerpredict the turbulence levels, and underpredict the velocity peak- a physicaliy inconsistent result.

The trajectory of the jet as inferred from the velocity peaks in Fig. 7 is relatively insensitive to the differencing scheme employed. The excellent correspondance in the location of the velocity peaks indicates that the pressure field controls the penetration of the jet as was proposed by Refs. 2 and 18.

Midplane $(Z / D=2)$ experimental data on the axial velocity is avallable in Ref. 7 . However, there is not much to be learned from this data. The mainstream flow is simply accelerated between the jets resulting in a uniform, higher-thanmainstream velocity profile that matches well with the calculations. Nothing of interest occurs in this plots and, therefore, they are not shown.

Underpredicted levels of turbulence are also suggested from the jet concentration contours of Fig. 8. Both BSUOS2 and hybrid calculations display steeper concentration gradients and less lateral mixing than in the experimental contours. Although as in the velocity field, the trajectory of the jet appears to be well represented, the level of mixing is significantly underpredicted. It is interesting to note that the BSUDS2 calculations display less mixing than the hybrid results even though the BSUDS2 calculations displayed higher turbulence intensities. Evidently the improved numerical accuracy of BSUDS2 in calculating scalar transport (jet concentration) counteracts the higher calculated turbulence levels. The hybria results indicate that numerical inaccuracy is "swamping" the calculated turbulent diffusion.

Another factor involved in the calculation of scalar transport is the determination of the turbulent Schmidt number. Johnson et al. 22 have shown that turbulent Schmidt number can vary significantly in a complex flow. Numerical calculations almost never account for this factor. The calculations reported here utilized a constant value of $\sigma t=0.5$ which could be used as a fairly representative average of the variation measured in Ref. 23. If the velocity and turbulence fields were correctly calculated, one could say that the scalar field (jet concentration level) requires a level of closure accounting for the variation in turbuient Schmidt number. Unfortunately, all variables are to some extent incorrectly calculated and the most that can be said about the BSUDS2 results is that they are al1 consistent. 'In other words, for the BSUDS2 calculations the turbulence levels are underpredicted, velocity peaks are too great, and the concentration contours are too steep. A similar consistent pattern is not evident in the hybrid calculations.

Closely spaced jets, $S / D=2$. The centerline jet velocity profiles for the closely spaced jet calculations are shown in Fig. 9. The distortion caused by the crossflow is greater than in widely spaced jet calculations ( $F i g .5)$. The mainstream flow exerts more of a force on the jet since the closer spacing of the jets restricts the flow area to which the flow may be diverted around the jet. The effect of this velocity distortion is to change the penetration angle of the jet as it leaves the outlet.

This change in the penetration angle of the jet alters its trajectory further downstream. As displayed in Fig. 10(a) the velocity distortion results in the jet trajectory being underpredicted for both hybrid and BSUDS2 calculations. (Here, as earlier, the velocity peak around $Y / D=2.81 \mathrm{~s}$ used as an indication of the jet trajectory.) In Fig. 10(b) the results of a calculation where the jet was defined at the outlet plane with a uniform, undistorted velocity profile are displayed.* (This is the only figure in which uniform inlet conditions at the wall were specified.) Using this boundary condition the jet trajectory is correctly predicted. Examined together, these calculations display the sensitivity of the jet trajectory to the specification of the jet boundary condition.

One trend unaffected by the change in boundary conditions is the overprediction of the magnitude of the velocity peak with BSUDS2. This trend is similar to the widely spaced jet calculations and is due to the same numerical accuracy considerations noted earlier.

Midplane axial velocity profiles are shown in Fig. 11. In this figure neither the BSUDS2 or the hybrid calculations display the velocity defect (wake) that was measured experimentally. This result may be physically consistent with an underprediction of the lateral turbulence intensity. In other words, if the lateral turbulence intensity is underpredicted, the jet will not spread in the lateral direction as rapidly as it does in the experiment. This might result in the velocity

*External to the jet supply pipe, equivalent computational meshes were used to eliminate grid dependency as a factor in the displayed comparison. 
defect not being "felt" at the midplane. Another explanation for the observed difference may be due to experimental error, as will be discussed in the final paragraph of this section.

The jet concentration contours for the closely spaced jets are shown in Fig. 12.** The caiculated contours again exhibit steeper gradients than the experimental values as was seen in the $S / D=4$ case, (Fig. 8). The trajectory of the jet fluid is underpredicted as was the velocity field, (Fig. 10(a)).

While the pressure field apparently controls the pentration of the widely spaced jets, this is less clear in the closely spaced jet calculations. When a supposedly more physically appropriate boundary condition is imposed (by specifying the velocities two jet diameters upstream of the orifice outlet) the jet trajectory is poorly predicted. Arbitrarily imposing a uniform velocity profile at the jet outlet improves the predicted trajectory, but for no physically correct reason.

A likely explanation for this discrepancy in the predicted trajectory may be experimental error. Khan indicates that his mainstream flow may have been slightly low for some of his measurements. This would aiter the jet-to-mainstream velocity ratio and, thereby, effect the penetra$t i o n$ of the jet. The discrepancy seen in the calculations is consistent with this type of experimental error. This error appears to be more significant for the closely spaced geometry than for the widely spaced geometry. The axial velocity profiles in Figs. 10 and 11 (closely spaced geometry) somewhat indicate a mass flow discrepancy. The computer program fully conserves mass, hence, any mass balance variation must be due to experimental error. A similar mass flow discrepancy was not observed in the widely spaced geometry.

\section{Summary of Results}

A series of calculations of two geometries, closely spaced and widely spaced jets, were made using two alternate convective differencing schemes and compared to experimental data. From this study the following points were suggested:

1. The BSUDS2 calculations displayed much less numerical smearing than the hybrid calculations. Due to this improved accuracy, the BSUDS2 results were more physically consistent than the hybrid results.

2. External to the jet outlet, in the highly turbulent regions of the flow freid, the BSUDS2 calculations displayed steeper velocity gradients resulting in improved agreement with experimentally measured turbulence intensities.

**In the experiment only one jet of the row was supplied with a tracing gas. This results in the tracing gas diffusing beyond the flow planes of symmetry. This problem appears to be of minor importance in the widely spaced geometry, but it becomes significant at the low concentration levels for the closely spaced geometry. For this reason only the higher levels of jet concentration are shown in Fig. 12.
3. The jet trajectory was generally well predicted for the widely spaced geometry $(S / D=4)$, but was underpredicted for the closely spaced geometry $(S / D=2)$. This underprediction may be tracable to either experimental error or to the specification of the jet boundary condition.

\section{Concluding Remarks}

The BSUDS2 differencing has been shown in these calculations to provide results that are more accurate than hybrid differencing. While the BSUDS2 results are not shown to be gridindependent, this is unilkely to significantly effect the reported trends. Additional grid refinement would probably only slightly change the BSUDS2 results. With this caveat, it appears that some conclusions may be drawn concerning the turbulence model. The $k-\varepsilon$ turbulence model appears to provide only limited usefuiness in the flow fields studied here. The isotropy assumption embodied in the $k-\varepsilon$ model is inconsistent with experimental data. An algebraic stress model may improve the deficiencies noted in the anisotropic region of the flow, thereby, improving the calculated mixing of the jet.

\section{Acknowledgments}

Dr. Saadat Syed of United Technologies, Pratt and Whitney Aircraft Group, made many useful comments which have been incorporated into this report.

\section{References}

1. Holdeman, J.D. and Srinıvasan, R., "On Modeling Dilution Jet Flowfields," NASA TM-83708, 1984 .

2. Kenworthy, M.J., Correa, S.M., and Burrus, D.L., "Aerothermal Modeling Program, Phase I, Vols. 1 and 2," General Electric Co., Cincinnat?, $\mathrm{OH}$, Nov. 1983. (NASA CR-168296).

3. Srinivasan, R., Reynolds, R., Ball, I., Berry, R., Johnsons, K., and Mongra, H., "Aerothermal Modeling Program Phase I, Vols. 1 and 2," Garrett Turbine Engine Co., Phoenic, AZ, GARRETT-21-4742-1, and -2, Aug. 1983. (NASA CR-168243).

4. Sturgess, G.J., "Aerothermal Modeling Phase I," Pratt and Whitney Aircraft Group, East Hartford, CT, PWA-5907-19, July 1983. (NASA CR-168202).

5. Patankar, S.V., Basu, D.K., and Alpay, S.A., "Prediction of the Three-Dimensional Velocity Field of a Deflected Turbuient Jet," Journal of Fluids Engineering, Vol. 99, No. 4, Dec. 1977, pp. 758-762.

6. Khan, Z.A., McGuirk, J.J., and Whitelaw, J.H., "A Row of Jets in Crossflow, "Fluid Dynamics of Jets with Applications to VISTOL, AGARD CP-308, 1982, Paper 14.

7. Khan, Z.A., "Opposed Jets in Crossflow," Phd Thesis, Mechanical Engineering Dept., Imperial College, University of London, 1982. 
8. Syed, S.A., Chrappetta, L.M., and Gosman, A.D., "Error Reduction Program," Pratt and Whitney Aircraft Group, East Hartford, CT, PWA-5928-25, 1985. (NASA CR-174776).

9. Launder, B.E., and Spaulding, D.B., "The Numerical Computation of Turbulent Flows," Computer Methods in Applied Mechanics and Engineering, Vol. 3, No. 2, Mar. 1974, pp. 269-289.

10. Raithby, G.D., "Skew-Upstream Differencing Schemes for Problems Involving Flurd Flow," Computer Methods in Applied Mechanics and Engineering, Vol. 9, No. 2, Oct. 1976, pp. 153-164.

11. Claus, R.W., Neely, G.M., and Syed, S.A., "Reducing Numerical Diffusion for Incompressible Flow Calculations," NASA TM-83621, 1984.

12. Boris, J.P. and Book, D.L., "Flux-Corrected TYransport. 1. SHASTA, A Fluid Transport Algerithm That Works," Journal of Computational Physics, Vol. II, No. 1, Jan. 1973, pp. 38-69.

13. Patankar, S.V., Numerical Heat Transfer and Fluid Flow, Hemisphere Publishing Corp., New York, 1980.

14. Issa, R.I., "Numerical Methods for Two- and Three-Dimensional Recirculating Flows," Computational Methods for Turbulent, TranSonic, and Viscous Flows, J.A. Essers, ed., Hemisphere Publishing, New York, 1983, pp. 183-212.

15. Neely, G.M., and Claus, R.W., "Accelerated Convergence for Incompressible Flow Calculations," NASA TM-86863, 1985.
16. Andreopoulos, J., "Measurements in Pipe Flow Issurng Perpendicularly Into a Cross Stream," Journal of Fluids Engineering, Vol. 104, No. 4, Dec. 1982, pp. 493-499.

17. Holdeman, J.D., and Srinivasan, R.," Experiments in Dilution Jet Mixing-Effects of Multiple Rows and Noncircular Orifices," AIAA Paper 85-1104, 1985.

18. Crabb, D., Durao, D.F.G., and Whitelaw, J.H., "A Round Jet Normal to a Crossflow," Journal of Fluids Engineering, Vol. 103, No. I, Mar. 1981, pp. 142-153.

19. Claus, R.W., "Analytical Calculation of a Single Jet in Crossflow and Comparison With Experiment," NASA TM-83027, 1983.

20. Roback, R., and Johnson, B.V., "Mass and Momentum Turbulent Transport Experiments with Confined Swirling Coaxial Jets, "United Technologies Research Center, East Hartford, CT, R83-915540-26, Aug. 1983. (NASA CR-168252).

21. So, R.M.C., Ahmed, S.A., and Mongra, H.C., "An Experimental Investigation of Gas Jet's in Confined Swirling Air Flow," Arizona State Univ., Tempe, AZ, Sept. 1984. (NASA (R-3832).

22. Johnson, B.V., Bennett, J.C., "Mass and Momentum Turbulent Transport Experiments with Conf ined Coaxial Jets," United Technologies Research CEnter, East Hartford, CT, R81-915540-9, Nov. 1981. (NASA CR-165574).

23. Johnson, B.V., Roback, R., and Bennett, J.C., "Scalar and Momentum Turbulent Transport Experiments With Swirling and Non-swirling rlcws," Experimental Measurements and Techniques in Turbulent Reactive and Non-Reactive Flows, AMD-Vol. 66, R.M.C. So, J.H. Whitelaw, and M. Lapp, eds., ASME, New York, 1984, pp. 107-120. 


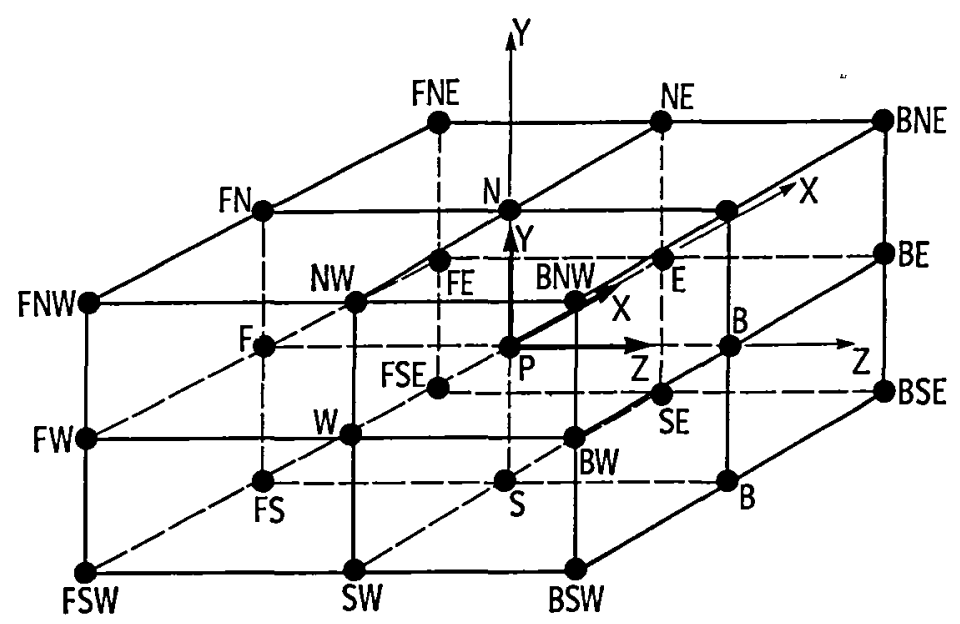

Figure 1. - Computational grid system for three dimensional calculations.

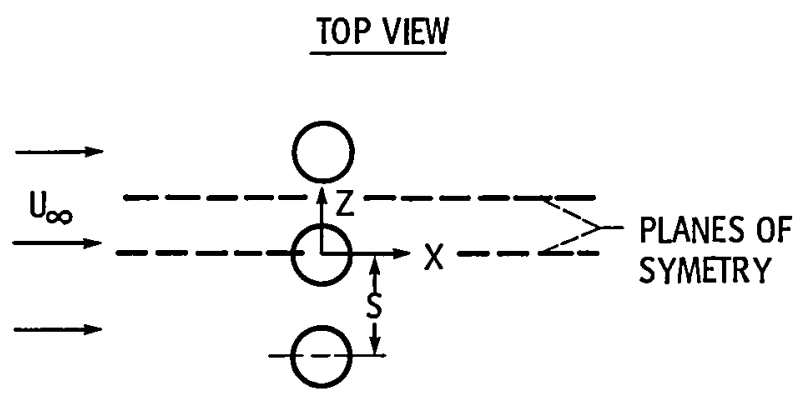

SIDE VIEW

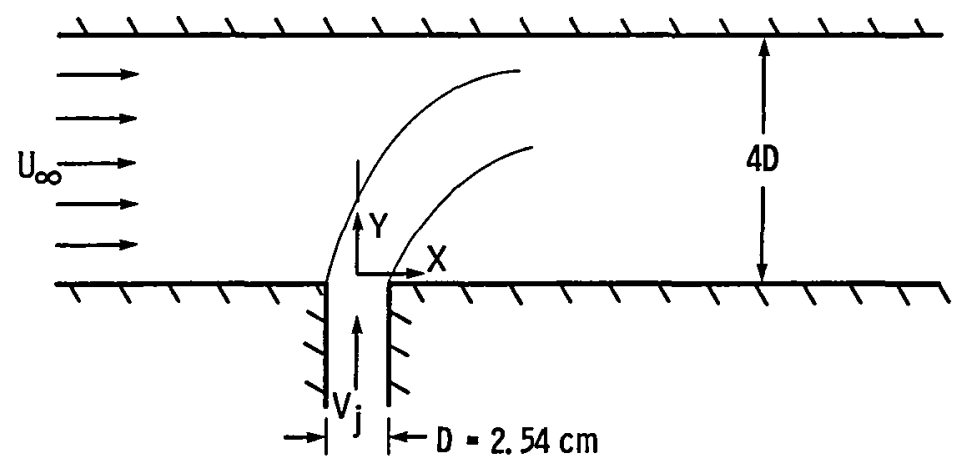

Figure 2. - Jet geometry coordinate system. 


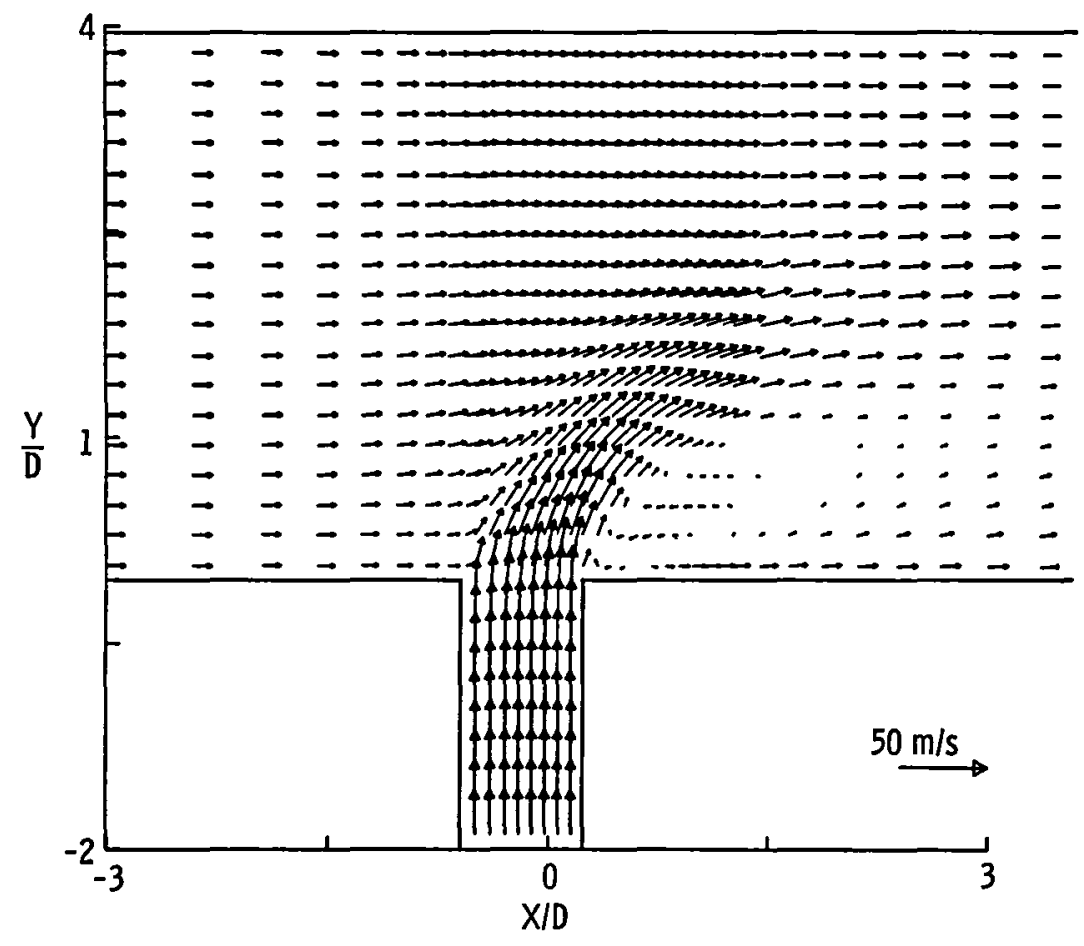

Figure 3. - Velocity vectors displaying grid node clusterıng near jet outlet. Vectors are shown for a portion of the calculated flow field near the jet centerline $(Z / D=0.25)$.

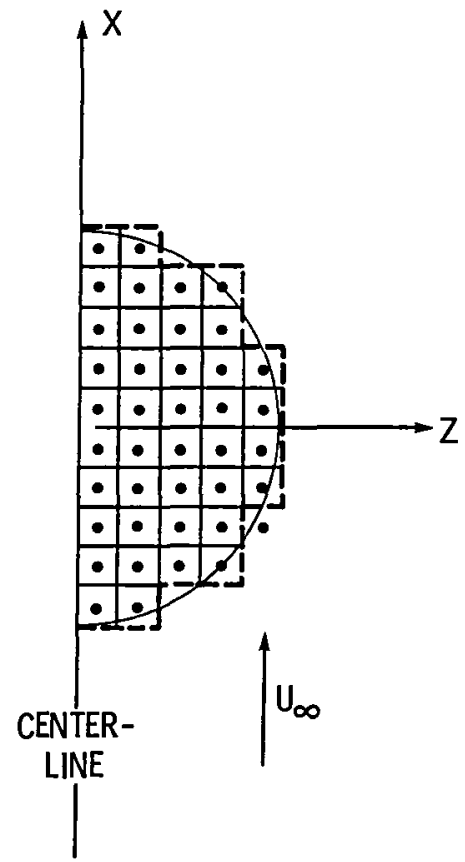

Figure 4. - Grid structure used to approximate the circular jet outlet. 

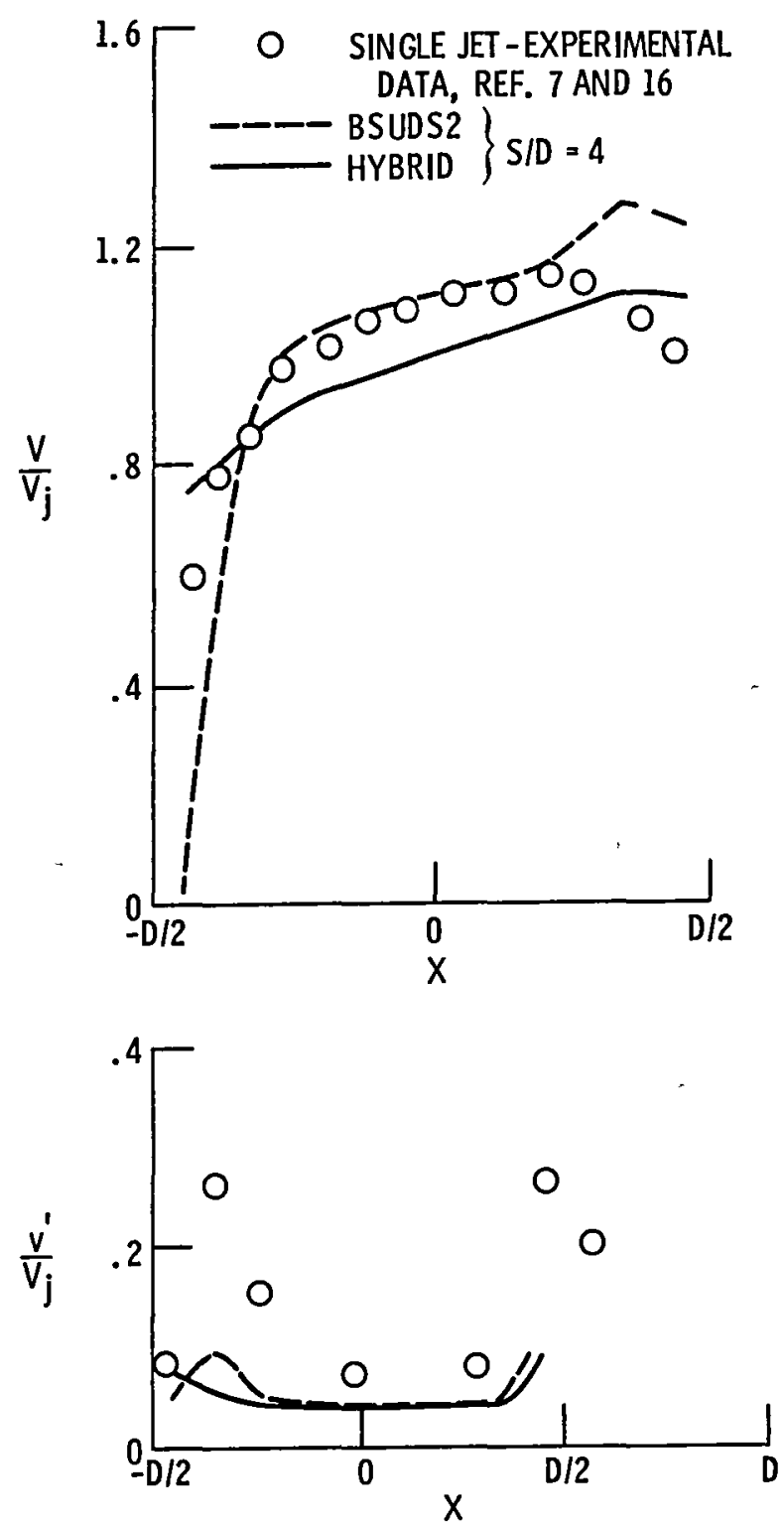

Figure 5. - Velocity and turbulence intensity at jet orifice along centerline $(Y / D=0$,

$Z / D=0$ ). Widely spaced jet geometry, $S / D=4$. 


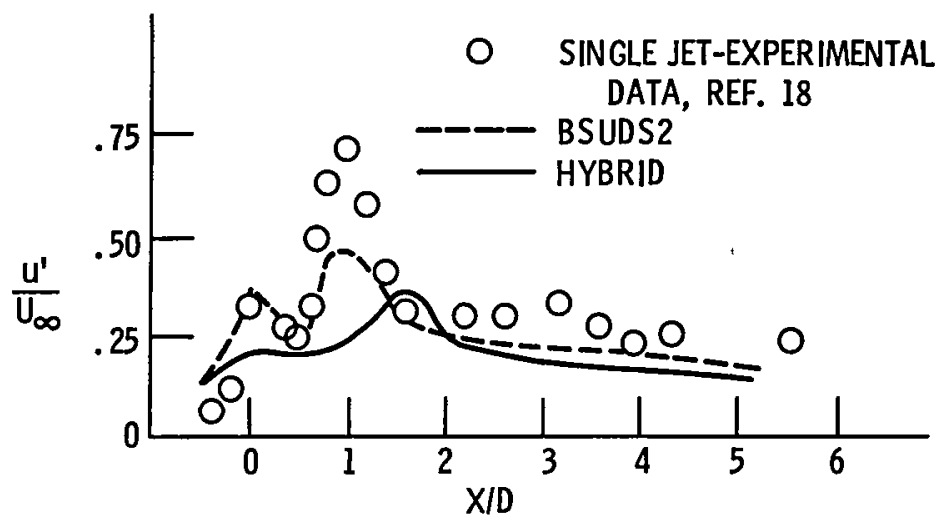

Figure 6. - Turbulence intensity along jet centerline $(Y / D=1.35, Z / D=0)$. Widely spaced jet geometry, $S / D=4$. 


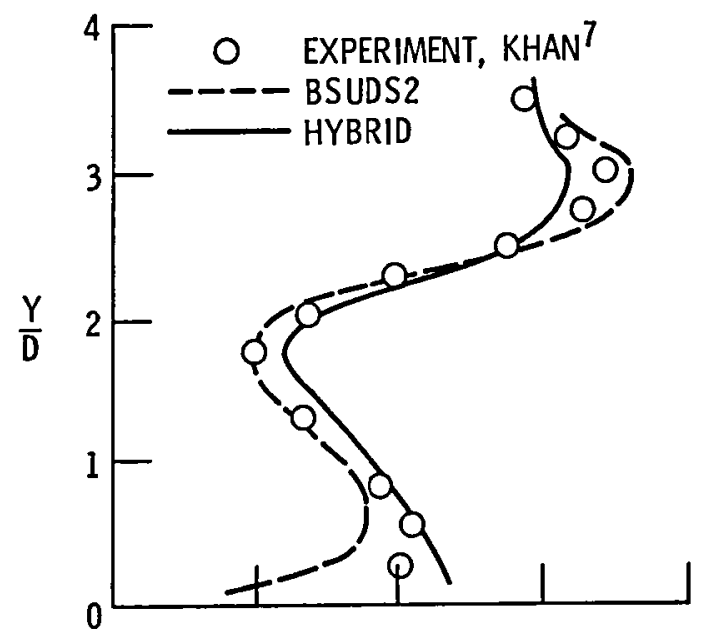

(a) $X / D=4$.

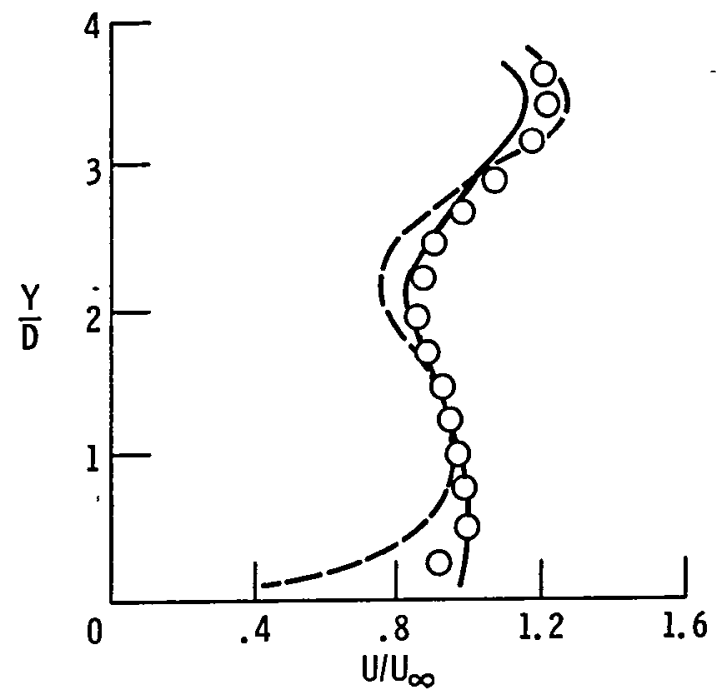

(b) $X / D=8$.

Figure 7. - Centerline axial velocity profiles attwo axial locations along jet centerplane $Z / D=0)$. Widely spaced jet geometry, $S / D=4$. 


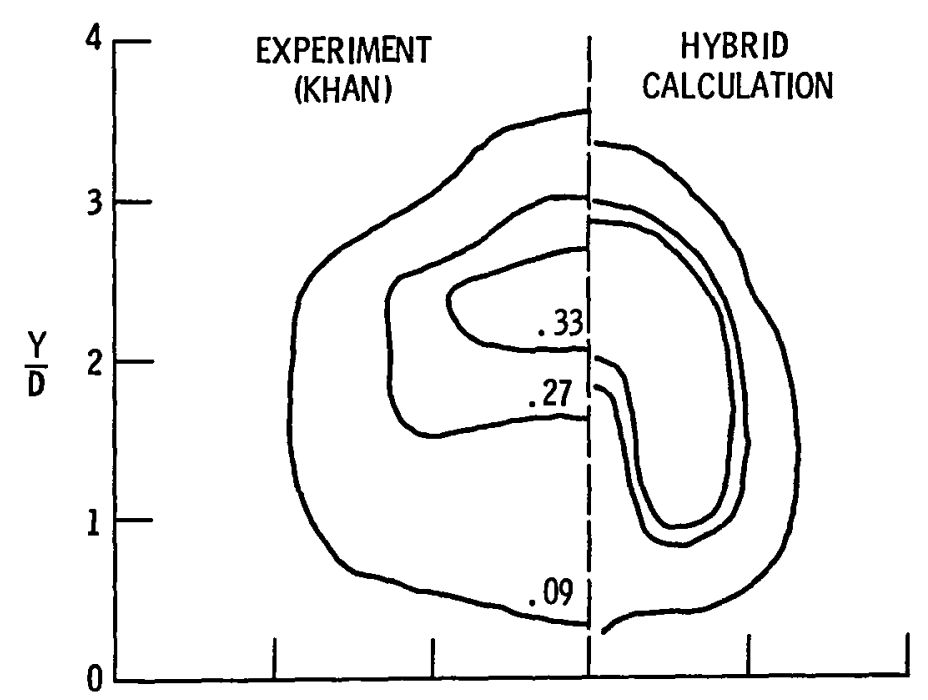

(a) Hybrid and experiment comparison.

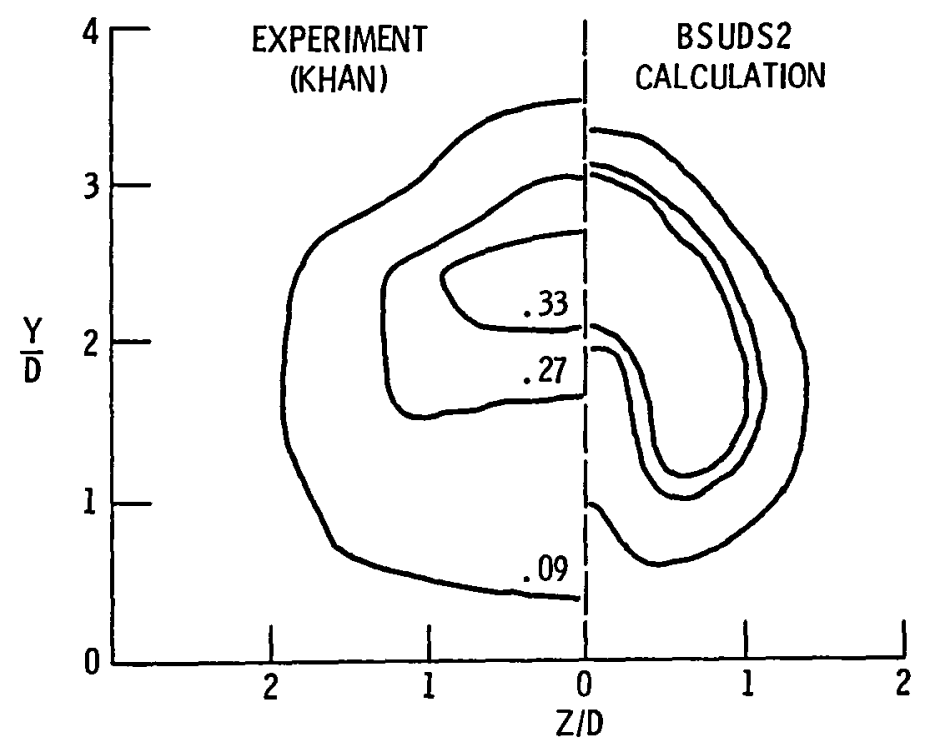

(b) BSUDS2 and experiment comparison.

Figure 8. - Jet concentration contours for $S / D=4$ at $X / D=4$. Concentration defined as: mainstream fluid $=0$, jet fluid $=1$. 


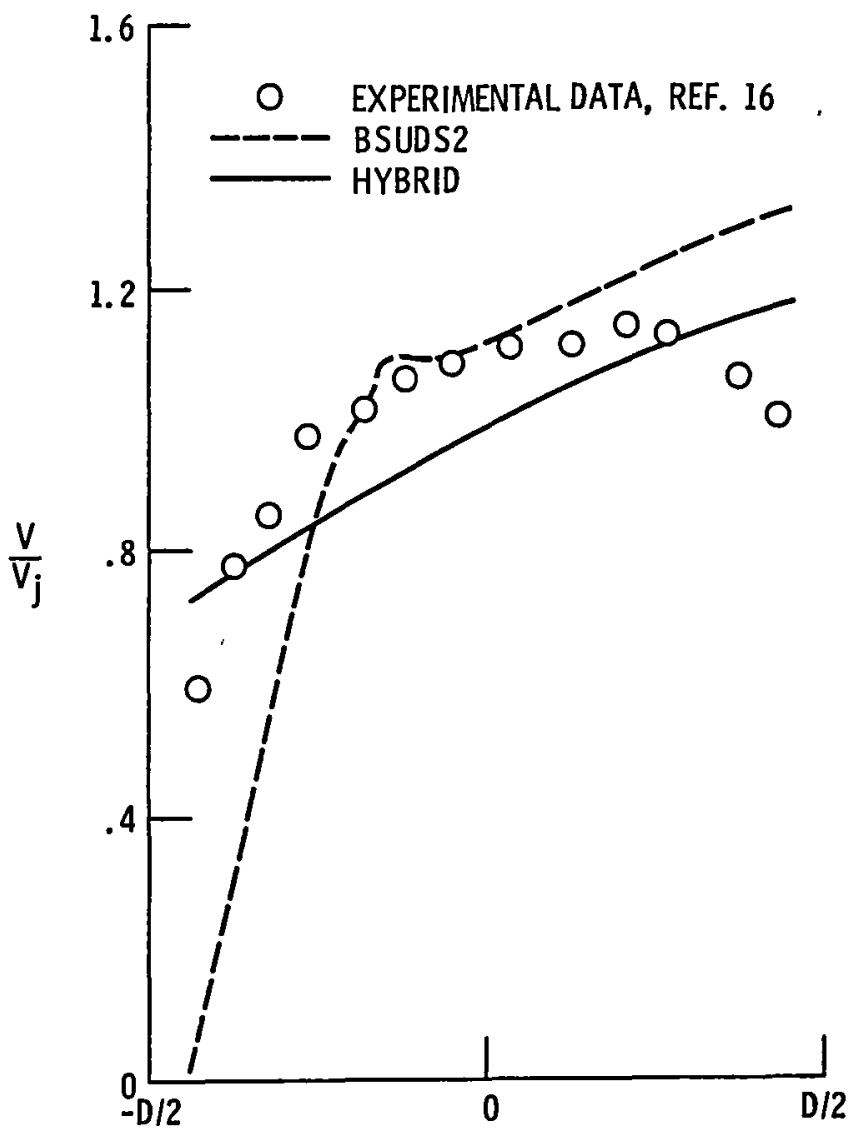

Figure 9. - Jet velocity profiles at the jet outlet along the centerline $Z / D=0)$. Calculations are for the closely spaced, $S / D=2$, geometry. 


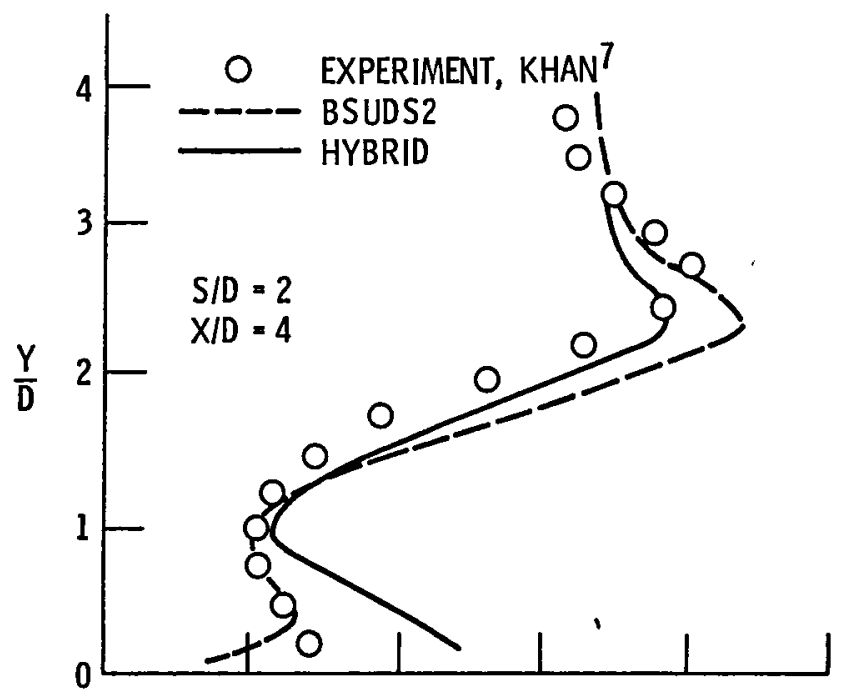

(a) Standard inflow boundary conditions with uniform injection at $Y / D=-2$.

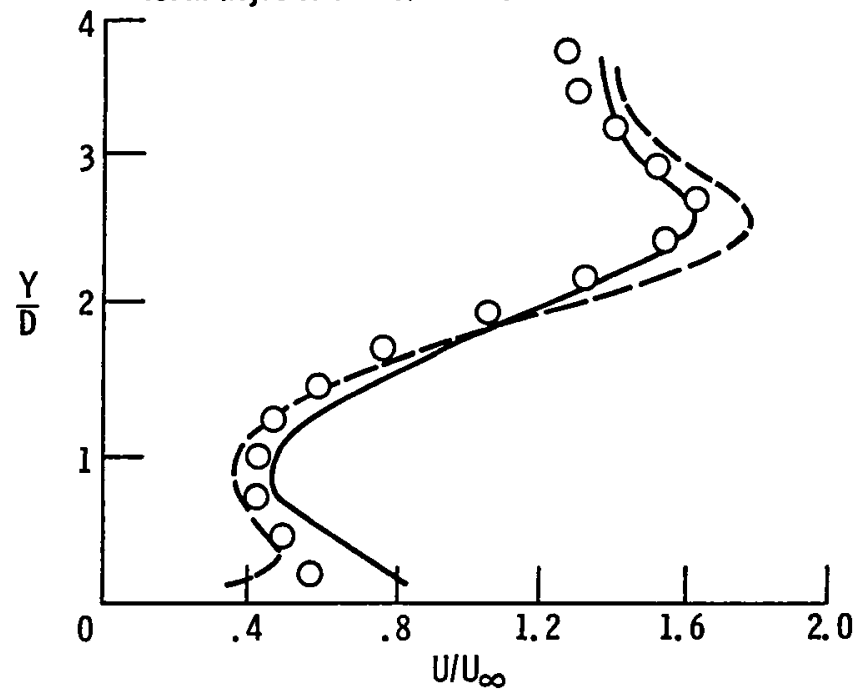

(b) Non-standard inflow boundary conditions with uniform in jection at outlet, $Y / D=0$.

Figure 10. - Centerline axial velocity profiles at one axial location, $X / D=4(Z / D=0)$. Closely spaced geometry, $S / D=2$. 


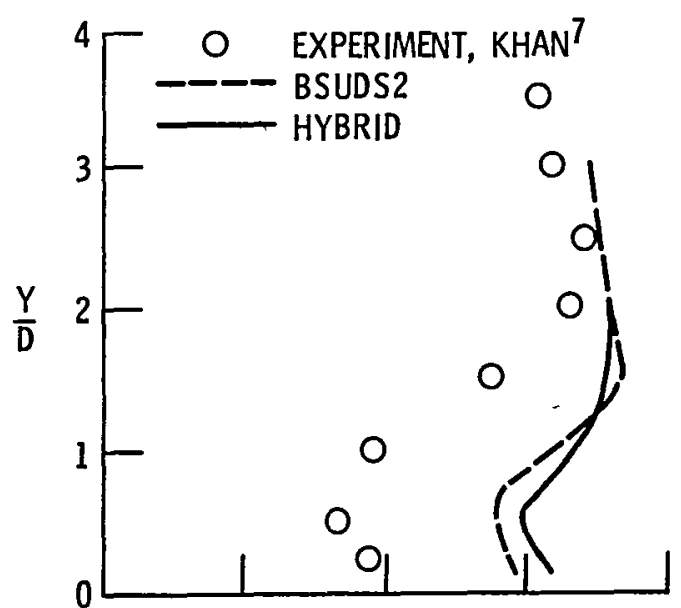

(a) $X / D=4$.

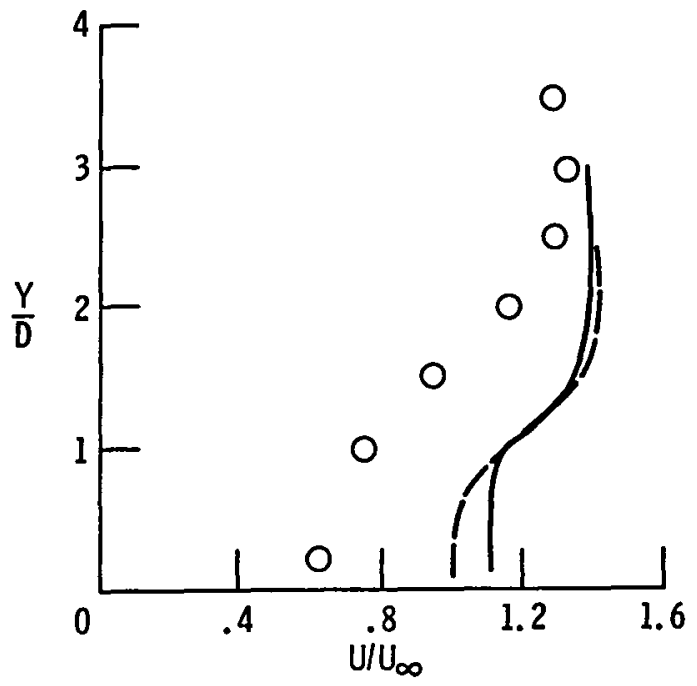

(b) $X / D=8$,

Figure 11. - Midplane axial velocity profiles at two axial locations $(Z / D=1)$. Closely spaced jet geometry, $S / D=2$. 


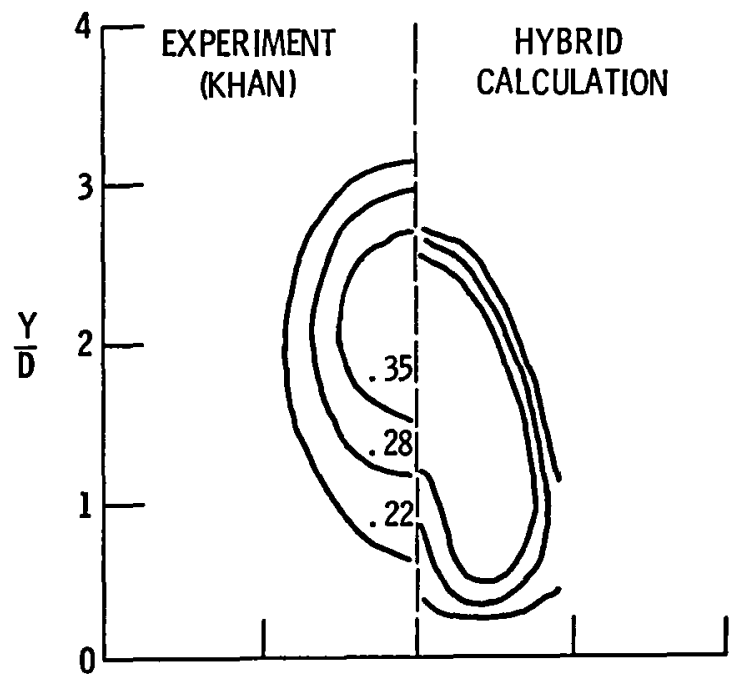

(a) Hybrid and experiment comparison.

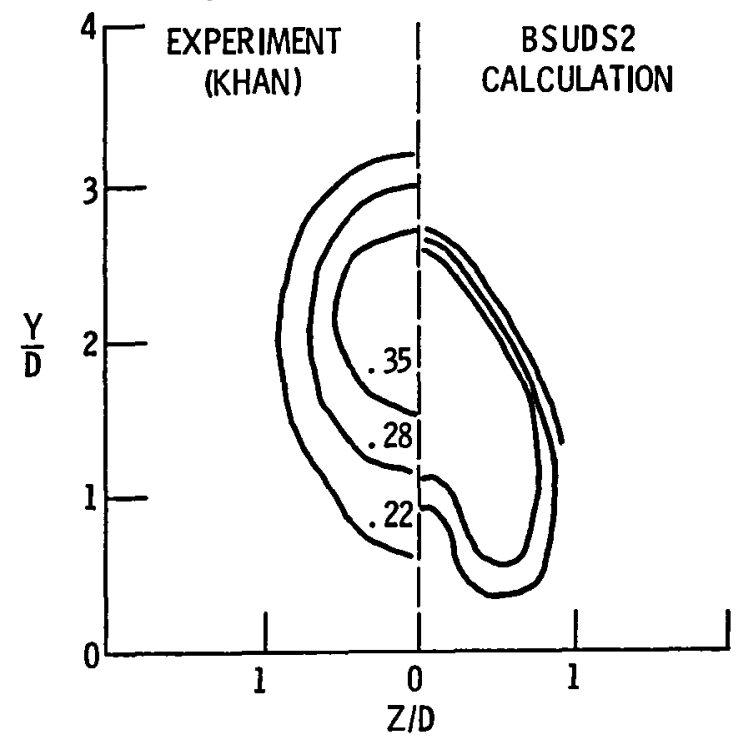

(b) BSUDS2 and experiment comparison.

Figure 12. - Jet concentration contours for $S / D=2$ at $X / D=4$. Concentration defined as: mainstream fluid $=0$, jet fluid $=1$. 


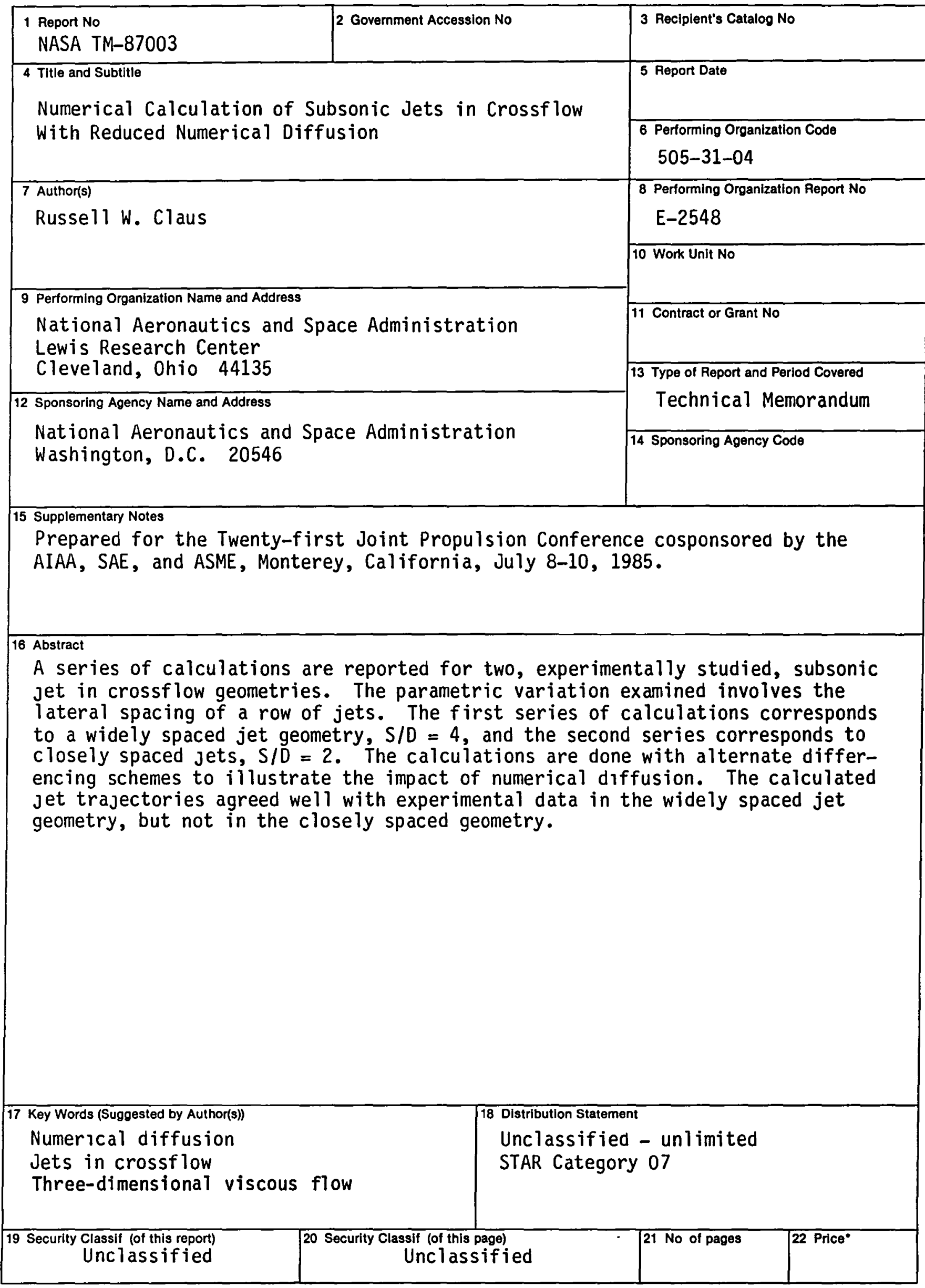

*For sale by the Natıonal Technical Information Service, Springfield, Virginia 22161 


\section{End of Document}

